s'effectuer à de grandes distances. C'est là une opération dōnt je me suis rendu coupable, directement ou indirectement, bien des fois. Je dis coupable, parce que j'ai modifié les composantes fauniques de divers milieux, ce qui est généralement mal vu par la science qui s'appelle la Zoogéographie.

Arrêtons ici ces considérations générales préliminaires qui 'ont pour objet de nous pénétrer de l'importance des Trichoptères, par conséquent de la nécessité de les mieux connaître, puisqu'ils constituent l'une des composantes fondamentales de la sitèse. Comme pour les autres organismes qui ont déjà fait l'objet de notes documentaires que l'on a bien voulu trouver dignes d'intêrêt, nous dirons, ce que sont les Trichoptères, quels sont leurs genres de vie, quelles eaux ils fréquentent, pourquoi il faut en avoir chez soi le plus possible, en vue du Poisson le meilleur possible.

(A suivre.)

\title{
LES ARÊTES DANS LE POISSON D'ÉTANG
}

\author{
Par M. HIRSCH \\ Inspecteur des Eaux et Forkts en retraite.
}

Dans un rapport, dont la traduction a été publiée ici (r), M. le $\mathrm{D}^{\mathbf{r}}$ Emile UNGER a fait très justement ressortir les inconvénients de certaines arêtes de poissons d'eau douce au point de vue culinaire. Il convient de noter la distinction très exacte qu'il fait entre les arêtes fines " sortes d'aiguillons qui se rencontrent dans la musculature ", dépourvues de calcium, et les arêtes principales constituant le squelette osseux de l'animal.

Mais les remèdes qu'indique ce savant nous paraissent constituer une assez médiocre propagande en faveur de la consommation du poisson d'eau douce. Déclarer en effet au consommateur qu'il nè doit en manger qu'à condition, soit de hacher menue la chair avant la cuisson, soit d'en faire une soupe passée au tamis; soit encore en friture, ce qui est alors plus acceptable, et que ce consommateur doit renoncer, sous peine d'étranglement, aux succulentes recettes publiées par l'U. $N$. S. E., il ne nous parait pas qu'une telle propagande soit vraiment de nature à pousser les ichthyophages vers le poisson d'eau douce; ils se rejetteront sur les poissons de mer avec lesquels ils ne courent pas de risque d'étranglement.

Les carpiculteurs français abordent le problème à un tout autre point de vue : ils se sont préoccupes de supprimer les arêtes fines, ou tout au moins,

(1) Voir Bulletin : $-\mathrm{n}^{0} 115$, Juillet-Aout 1938, p. 13. 
si cette suppression totale s'avère impossible, de réduire leur nombre et de les rendre plus facilement décelables.

L'expérience a prouvé que ce n'est pas là une utopie : nombre d'élevages en France produisent des Carpes se rapprochant de bien près du but final.

Comment est-on parvenu à ce résultat ? Il semble qu'une explication puisse en être donnée, mais elle n'a rien de scientifique et procède sculement d'un certain nombre d'observations qui auraient besoin d'être contrôlées.

Deux facteurs principaux apparaissent :

$I^{\circ}$ Sélection ;

$2^{\circ}$ Nourriture.

$I^{\circ}$ Sélection. - Il ne semble guère que nous ayions à nous arrêter à la sélection : tous nos lecteurs savent que, par sélection, on parvient à obtenir de sérieuses modilications de forme el de constitution des animaux. Personnellement, j'ai profité d'une sélection préparée de longue date par nos amis Tchéco-Slovaques, et leur race s'est parfaitement acclimatée dans les étangs du Centre. Nous avons, mon regretté ami Meugniot et moi, fait choix de cette race de préférence à d'autres ayant des facultés d'accroissement équivalentes ou mème supérieures, précisément parce que nous avions remarqué que la chair de ces Carpes, par ailleurs d'excellent goût, ne contenait qu une faible quantité d'arêtes fines ( $I$ ).

Nous n'avons donc eu qu'à perfectionner les résultats déjà obtenus, et c'est là qu'intervient, nous avons lieu de le penser, la question de nourriture.

$2^{\circ}$ Nourriture. - On observe que les Carpes de race parfaitement sélec tionnée, abandonnées dans des mares où elles ne trouvent qu'une pitance tout à fait insuffisante, maigrissent et prennent une forme allongée se rapprochant de la Carpe commune. En mème temps, on constate un développement considérable de fines arêtes en pleine chair : ce sont des arêtes menues, élastiques, invisibles, et parfaitement honnies, à juste titre, des dégustateurs.

La sélection n'est donc pas tout dans cette question : la nourriture a une influence incontestable.

Mais là encore, il y a nourriture et nourriture : on voit en effet, dans des étangs à Carpes sélectionnées, dans lesquels la nourriture, naturelle ou complétée par de la nourriture artificielle, est abondante, les arêtes fines continuer à se produire, en quantité moindre, il est vrai, mais néanmoins suffisante, pour ètre désagréables.

De nombreuses études ont été faites, notamment dans les pays de l'Europe Centrale, sur les qualités nutritives des divers aliments' ; mais ces étu-

(I) Voir Bulletin: - $\mathrm{n}^{\circ}$, Août ${ }_{1928}$, p. 3 . 
des n'ont porté, à ma connaissance, que sur le rendement de ces aliments par rapport au prix de revient; il ne semble pas que les laboratoires se soient jamais préoccupés de l'influence de certains aliments sur la production, ou inversement, l'absence d'arêtes fines.

C'est là pourtant une question primordiale au point de vue de la propagande, et ce problème mériterait des expériences méthodiques et suivies par les laboratoires.

Nos observations personnelles, purement empiriques, auraient besoin d'être contrôlées scientifiquement.

Nous avons remarqué que les Carpes, nourries avec un complément de céréales seules : maïs (très employé), blé, pâtes, etc., sont loin d'être exemptes de fines arêtes. Les Carpes que Meugniot et moi avions prises en TchécoSlovaquie avaient été nourries avec un complément de lupin. Pour mon compte, je n'ai jamais réussi à me procurer des tonnages suffisants de graines de lupin pour faire une nourriture d'élevage ; mais Mevgniot, plus heureux, a pu faire un essai pendant une année, et il avait cru constater une diminution des arêtes fines.

La caractéristique de la graine de lupin est sa haute teneur en protéïne, tandis que le maïs est riche, trop riche même pour le poisson, en matières amylacées.

Nous avons été conduits à distribuer une nourriture complémentaire d'autant plus azotée que le poisson à nourrir est jeune ; c'est en somme basé sur le même principe qui fait qu'on s'attache à accroitre le plancton dans les étangs de pose, que l'on garnit souvent de fumier frais. Nous donnons du sang presque pur au frai venant d'éclore; ensuite le sang cuit ou le tourteau d'arachide rufisque permettent' de doser la teneur en azote à ajouter.

Pour des raisons de commodités locales, nous avons surtout utilisé le sang cuit pour le jeune frai. L'expérience a été favorable et a montré que le sang, riche à la fois en azote et en phosphates assimilables, développe avantageusement le squelette de l'animal en même temps que la musculature.

Lorsque nous avons soumis ces sujets fortement ossifiés à une nourriture moins riche, en vue de l'engraissement, il s'est trouvé que les arêtes fines étaient rares, et, ce qui d'ailleurs était à prévoir, grandes et solides, bien visibles : ainsi, le consommateur n'éprouve aucune difficulté à les retirer.

En résumé, voici la recette qui paraît recommandable au point de vue des arêtes fines :

Au début de l'existence du poisson (première enfance $=$ frai) sang sur support farineux, le tout bien cuit ;

Dans la jeunesse (alevins du second été) grains cuits avec un peu de sang ou mélangé de tourteau d'arachide rufisque ;

Pour les adultes propres à l'engraissement, en vue de faire du poisson marchand, grains cuits avec un peu de tourteau. 
Les grains qui paraissent avoir donné les meilleurs résultats sont ceux de vesce, dont la valeur en protéïne se rapproche de celle du lupin.

Etant données les difficultés à se procurer le lupin ou la vesce, les Syndicats de carpiculteurs ne devraient pas se désintéresser de la culture de ces végétaux, car, pour eux, produire des poissons d'étangs, sans fines arêtes, est bien un nœud de leur propagande.

Signalons en passant l'effet de cette nourriture sur les Brochets ; ceux-ci, carnivores, ne l'absorbent qu'indirectement par les poissons qu'ils dévorent. Depuis plusieurs années, les Brochets de notre élevage, d'origine indigène et non soumis à une sélection spéciale, n'ont, eux aussi, que de rares fines arêtes, mais guère fines, car robustes et faciles à retirer de la chair cuite. On a même pu, dans de jeunes Brochets, retirer des filets comme à une sole et sans y trouver aucune arête.

Il semble résulter de ces faits que, pour produire du poisson d'étang sans arêtes fines, et surtout sans fines arêtes, il convient de leur constituer une ossature robuste, servant de charpente solide sur laquelle s'appuie la musculature, puis développer cette musculature par une nourriture appropriée à l'âge des sujets, les arêtes fines ne se développant que pour soutenir une chair elle-même insuffisamment soutenue par les muscles trop faibles.

Il est regrettable que les laboratoires n'aient pas encore procédé à des expériences méthodiques sur ce sujet, de façon à savoir si cette théorie, purement empirique, est confirmée en tout ou partie, et si l'on peut en tirer des conclusions pratiques pour faire connaître aux producteurs les moyens les plus appropriés selon les circonstances particulières, pour qu'ils arrivent à réduire, dans toute la mesure possible, ces arêtes fines si préjudiciables au développement de la consommation du poisson d'étang.

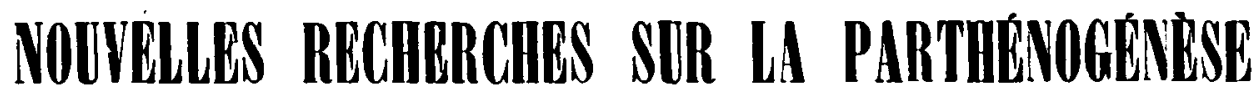 ARTIFICIRLLL DES POISSONS
}

\author{
Par M. Edouard PIROLA \\ Directeur de l'Institut de Pisciculture de Bellano (Italie).
}

Durant la campagne de I $^{3} 3_{7}-1938$, j'ai continué persévéramment mes travaux sur la parthénogénèse artificielle des poissons d'eau douce dont le premier compte rendu remonte à 1933 (I). Durant l'hiver et le printemps,

(1) Voir Bulletin : - $n^{\circ} 64$, Octobre 1933 , p. $101 ;-n^{\circ} 65$, Novembre 1933 , p. 135 ; $-n^{\circ} 7^{2}$, Juin $193 \hat{4}$, p. $32 \mathrm{x} ;-n^{\circ} 79$, Janvier 1935, p. $165 ;-n^{\circ}$ 105, Mars $19^{3} 7$, p. 224 